Pacific Journal of Mathematics

A CHARACTERIZATION OF PRÜFER DOMAINS IN TERMS OF

ROBERT WiLLIAM GILMER, JR. AND JOSEPH F. HOFFMAN 


\title{
A CHARACTERIZATION OF PRÜFER DOMAINS IN TERMS OF POLYNOMIALS
}

\author{
Robert Gilmer and Joseph F. HoffmanN
}

\begin{abstract}
Assume that $D$ is an integral domain with identity and with quotient field $K$. Each element of $K$ is the root of a polynomial $f$ in $D[X]$ such that the coefficients of $f$ generate $D$ if and only if the integral closure of $D$ is a Prüfer domain.
\end{abstract}

All rings considered in this paper are assumed to be commutative and to contain an identity element. By an overring of a ring $R$, we mean a subring of the total quotient ring of $R$ containing $R$. The symbol $X$ in the notation $R[X]$ denotes an indeterminate over $R$.

In the study of integral domains, Prüfer domains arise in many different contexts. See, for example, [1; Exer. 12, p. 93] or [2; Chap. IV] for some of the multitudinous characterizations of Prüfer domains. Among such characterizations there are at least two in terms of polynomials: (1) The domain $D$ is a Prüfer domain if and only if $A_{f} A_{g}=A_{f g}$ for all $f, g \in D[X]$, where $A_{h}$ denotes the ideal of $D$ generated by the coefficients of the polynomial $h \in D[X]\left(A_{h}\right.$ is called the content of $h$ ) [3], [10], [2; p. 347]. (2) $D$ is a Prüfer domain if and only if $D$ is integrally closed and for each prime ideal $P$ of $D$, the only prime ideals of $D[X]$ contained in $P[X]$ are those of the form $P_{1}[X]$, where $P_{1}$ is a prime ideal of $D$ contained in $P[2 ; \mathrm{p} .241]$. In Theorem 2 we provide another characterization of Prüfer domains in terms of polynomials: $D$ is a Prüfer domain if and only if $D$ is integrally closed and each element of the quotient field $K$ of $D$ is a root of a polynomial $f \in D[X]$ such that $A_{f}=D$. Then in Theorem 5 we obtain an extension of this result to the case where $D$ need not be integrally closed.

Our interest in domains $D$ such that each element of $K$ is a root of a polynomial $f \in D[X]$ with $A_{f}=D$ stemmed from the fact that this property is common to both $\Delta$-domains - that is, integral domains whose set of overrings is closed under addition [4] - and to integral domains having property $(n)$ for some $n>1$ - that is, integral domains $D$ with the property that $(x, y)^{n}=\left(x^{n}, y^{n}\right)$ for all $x, y \in D$ [9]. Thus, if $D$ is a $\Delta$-domain with quotient field $K$ and if $t \in K$, then since $D\left[t^{2}\right]+D\left[t^{3}\right]$ is an overring of $D, t^{5}=t^{2} t^{3} \in D\left[t^{2}\right]+D\left[t^{3}\right]$, whence it is evident that $t$ is the root of a polynomial in $D[X]$ in which the coefficient of $X^{5}$ is a unit. If $D$ has property $(n)$ for some $n>1$ and if $t=a / b \in K$, where $a, b \in D$ and $b \neq 0$, then from the equality $(a, b)^{n}=$ $\left(a^{n}, b^{n}\right)$ it follows that $a^{n-1} b=d_{1} a^{n}+d_{2} b^{n}$ for some $d_{1}, d_{2} \in D$; divid- 
ing both sides of this equation by $b^{n}$ yields $d_{1} X^{n}-X^{n-1}+d_{2}$ as a polynomial satisfied by $t$.

We show that the condition described in the preceding paragraph is equivalent to the condition that each element of the quotient field of $D$ satisfies a polynomial with a unit coefficient.

THeOREM Let $f=\sum_{i=0}^{n} f_{i} X^{i}$ be an element of $R[X]$. Then $A_{f}=$ $\left(f_{0}, f_{1}, \cdots, f_{n}\right)$ is the set of coefficients of elements of the principal ideal of $R[X]$ generated by $f$.

Proof. Denote by $E$ the set of coefficients of elements of $(f) ; E$ is an ideal of $R$ and the inclusion $A_{f} \supseteq E$ is clear. Conversely, if $t=\Sigma_{0}^{n} r_{i} f_{i}$ is an element of $A_{f}$, then $\left(\sum_{i=0}^{n} r_{i} X^{n-i}\right) f$ is an element of $(f)$ and the coefficient of $X^{n}$ in this polynomial is $t$. Hence $t \in E$ and the equality $E=A_{f}$ holds, as asserted.

A modification of the proof of Theorem 1 shows that the result generalizes to polynomials in an arbitrary set of indeterminates, and this observation, in turn, yields a further generalization of Theorem 1.

COROLlaRY 1. Let $\left\{f_{\alpha}\right\}$ be a subset of the polynomial ring $R\left[\left\{X_{\lambda}\right\}\right]$, and for each $\alpha$, let $A_{f_{\alpha}}$ be the ideal of $R$ generated by the coefficients of $f_{\alpha}$. Then $\Sigma_{\alpha} A_{f_{\alpha}}$ is the set of coefficients of the ideal of $R\left[\left\{X_{\lambda}\right\}\right]$ generated by $\left\{f_{\alpha}\right\}$.

The equivalence of the two conditions mentioned in the paragraph immediately preceding Theorem 1 also follows at once from this result. If $S$ is a unitary extension ring of $R$, we say that $R$ has property $(P)$ with respect to $S$ or that $S$ is a P-extension of $R$ if each element of $S$ satisfies a polynomial in $R[X]$ one of whose coefficients is a unit of $R$, or, equivalently, whose coefficients generate the unit ideal of $R$. The next result is not unexpected.

THEOREM 2. Let $D$ be an integrally closed domain with quotient field $K$. Then $D$ is a Prüfer domain if and only if $K$ is a P-extension of D.

Proof. If $D$ is a Prüfer domain, then $D$ has property $(n)$ for each positive integer $n$ [5; Theorem $2.5(\mathrm{e})$ ], [2; Theorem 24.3], and hence, as already shown, $D$ has property $(P)$ with respect to $K$. Conversely, suppose that $K$ is a $P$-extension of $D$. Let $M$ be a maximal ideal of $D$ and let $t$ be an element of $K$. Then $t$ is a root of a polynomial $f$ in $D[X]$ such that $A_{f}=D$, and hence $f \notin M[X]$. It then follows from [11; p. 19] that $t$ or $t^{-1}$ is in $D_{M}$. Consequently, $D_{M}$ is a valuation ring and $D$ is a Prüfer domain, as asserted. 
To obtain a characterization of domains $D$ for which $K$ is a $P$-extension of $D$, we introduce some useful notation. Let $R$ be a ring, let $\left\{M_{\lambda}\right\}_{\lambda \in \Lambda}$ be the set of maximal ideals of $R$, and let $N$ be the set of elements $f$ in $R[X]$ such that $A_{f}=R ; \mathrm{W}$. Krull [7] observed that $N$ is a regular multiplicative system in $R[X]$ and he considered properties of the ring $R[X]_{N}$, which M. Nagata in [8; p. 17] denotes by $R(X) . \quad$ It is clear that $N=R[X]-\cup_{\lambda} M_{\lambda}[X]$, and in Chapter 33 of [2] it is shown that if an ideal $E$ of $R[X]$ is contained in $\cup_{\lambda} M_{\lambda}[X]$, then $E$ is contained in one of the ideals $M_{\lambda}[X]$. Consequently, $\left\{M_{\lambda}[X]\right\}$ is the set of prime ideals of $R[X]$ maximal with respect to not meeting $N$ and $\left\{M_{\lambda} R(X)\right\}$ is the set of maximal ideals of $R(X)$. With these facts recorded, we state and prove our next theorem.

THEOREM 3. Let $T$ be a unitary extension ring of the ring $R$ and let $S$ be the integral closure of $R$ in $T$.

(a) The ring $S(X)$ is integral over $R(X)$.

(b) If $T[X]$ is integrally closed, then $S(X)$ is the integral closure of $R(X)$ in $T(X)$.

Proof. (a): Let $\left\{M_{\alpha}\right\}_{\alpha \in A}$ and $\left\{M_{\beta}^{\prime}\right\}_{\beta \in B}$ be the sets of maximal ideals of $R$ and $S$, respectively. If $N=R[X]-\cup_{\alpha} M_{\alpha}[X]$ and $N^{\prime}=$ $S[X]-\cup_{\beta} M_{\beta}^{\prime}[X]$, then $R(X)=R[X]_{N}$ and $S(X)=S[X]_{N^{\prime}} \quad$ The ring $S[X]_{N}$ is integral over $R[X]_{N}$ and we prove (a) by showing that $N^{\prime}$ is the saturation of the multiplicative system $N$ in $S[X]$. Let $N^{*}$ be the saturation of $N$ in $S[X]$; since $N \subseteq N^{\prime}$ and since $N^{\prime}$ is saturated, it follows that $N^{*} \subseteq N^{\prime}$. The multiplicative system $N^{*}$ is characterized as the complement in $S[X]$ of the set $\mathscr{P}$ of prime ideals of $S[X]$ maximal with respect to not meeting $N$; hence, to prove that $N^{\prime}$ is contained in $N^{*}$, we prove that $\mathscr{P} \subseteq\left\{M_{\beta}^{\prime}[X]\right\}_{\beta \in B}$. Thus, let $P^{\prime} \in \mathscr{P}$ and let $P^{\prime} \cap R[X]=P$. Since $P^{\prime} \cap N=\varnothing, P$ also fails to meet $N$ - that is, $P \subseteq \cup_{\alpha \in A} M_{\alpha}[X]$; as we remarked earlier, this inclusion implies that $P \subseteq M_{\alpha}[X]$ for some $\alpha \in A$. Since $S[X]$ is integral over $R[X]$, there is a prime ideal $Q^{\prime}$ of $S[X]$ such that $Q^{\prime}$ contains $P^{\prime}$ and $Q^{\prime} \cap R[X]=$ $M_{\alpha}[X]$. Hence $\left(Q^{\prime} \cap S\right) \cap R=\left(Q^{\prime} \cap R[X]\right) \cap R=M_{\alpha}[X] \cap R=M_{\alpha}$, a maximal ideal of $R$; from the integrality of $S$ over $R$ we infer that $Q^{\prime} \cap S$ is a maximal ideal of $S$, that is, $Q^{\prime} \cap S=M_{\beta}^{\prime}$ for some $\beta \in B$. It follows that $M_{\beta}^{\prime}[X] \subseteq Q^{\prime}$ and in fact, $Q^{\prime}=M_{\beta}^{\prime}[X]$ since $S[X]$ is integral over $R[X]$ and since $Q^{\prime} \cap R[X]=M_{\beta}^{\prime}[X] \cap R[X]=M_{\alpha}[X]$. We therefore obtain the inclusion $P^{\prime} \subseteq M_{\beta}^{\prime}[X]$. Since $M_{\beta}^{\prime}[X]$ misses $N$ and since $P^{\prime}$ is maximal with respect to missing $N$, it follows that $P^{\prime}=M_{\beta}^{\prime}[X]$ and $\mathscr{P} \subseteq\left\{M_{\beta}^{\prime}[X]\right\}_{\beta \in B}$. This completes the proof of (a).

To prove (b) we recall that $S[X]$ is the integral closure of $R[X]$ in $T[X]\left[2\right.$, Theorem 10.7], and hence $S[X]_{N}=S(X)$ is the integral closure of $R[X]_{N}=R(X)$ in $T[X]_{N}$. If $T[X]$ is integrally closed, then $T[X]_{N}$ 
is also integrally closed, and since $T(X)$ is an overring of $T[X]_{N}$, it follows that the integral closure of $R(X)$ in $T(X)$ coincides with the integral closure of $R(X)$ in $T[X]_{N}$. Thus $S(X)$ is the integral closure of $R(X)$ in $T(X)$, as asserted.

REMARK 1. The following result follows from the proof of part (a) of Theorem 3: Assume that $S$ is a unitary ring extension of the ring $R$ and that $S$ is integral over $R$. Let $N$ be a multiplicative system in $R$, let $\left\{P_{\alpha}\right\}$ be the set of prime ideals of $R$ maximal with respect to not meeting $N$, and let $\left\{P_{\beta}^{\prime}\right\}$ be the set of prime ideals of $S$ such that $P_{\beta}^{\prime} \cap R \in$ $\left\{P_{\alpha}\right\}$. Then $S-\left(\cup P_{\beta}^{\prime}\right)$ is the saturation of $N$ in $S$ (cf. [2; Proposition 11.10]). More generally, this conclusion is valid if the extension $R \subseteq S$ satisfies going up in the terminology of [6; p. 28].

REMARK 2. We do not know if the conclusion of (b) is valid without the hypothesis that $T[X]$ is integrally closed. As the proof of part (b) of Theorem 3 shows, sufficient conditions for $S(X)$ to be the integral closure of $R(X)$ in $T(X)$ are that $T[X]_{N}$ is integrally closed in $T(X)$, a quotient ring of $T[X]_{N}$. It is easy to give examples to show that the inclusion $T[X]_{N} \subseteq T(X)$ may be proper; if $R$ is a $v$-domain with quotient field $T$, then a necessary condition that $T(X)$ should be $T[X]_{N}$ is that $R$ be a Prüfer $v$-multiplication ring (see $\$ 33$ of [2] for terminology). The condition that $T[X]$ is integrally closed is not, insofar as we know, definitive in terms of $T$; it implies that $T$ is integrally closed, but the converse fails in general.

THEOREM 4. Assume that $T$ is a unitary extension ring of the ring $R$ and that $S$ is an intermediate ring integral over $R$. If $T$ is a $P$-extension of $S$, then $T$ is a P-extension of $R$.

Proof. Let $t \in T$, let $Q^{\prime}=\{f \in S[X] \mid f(t)=0\}$, and let $Q=$ $Q^{\prime} \cap R[X]$. If $N$ and $N^{\prime}$ are defined as in the proof of Theorem 3, so that $R(X)=R[X]_{N}$ and $S(X)=S[X]_{N^{\prime}}$, then the hypothesis that $T$ is a $P$-extension of $S$ implies that $Q^{\prime} \cap N^{\prime} \neq \varnothing$. If we show that $Q \cap$ $N \neq \varnothing$, then the proof of Theorem 4 will be complete. We first observe that $Q R(X)=Q^{\prime}(S[X])_{N} \cap R(X)$. That the right side contains the left side is clear, and if $f / n=d / m \in Q^{\prime}(S[X])_{N} \cap R(X)$, where $f \in Q^{\prime}$, $d \in R[X]$, and $n, m \in N$, then $f m=d n \in Q^{\prime} \cap R[X]=Q$, so that $f / n=f m / n m \in Q R(X)$ and $Q^{\prime}(S[X])_{N} \cap R(X)=Q R(X)$. It follows from the proof of Theorem 3 that $(S[X])_{N}=(S[X])_{N^{\prime}}$; hence

$$
Q R(X)=Q^{\prime} S(X) \cap R(X)=S(X) \cap R(X)=R(X),
$$

which means that $Q \cap N \neq \varnothing$. 
The characterization of Prüfer domains stated at the beginning of this paper is a direct consequence of the preceding results.

Theorem 5. Let $D$ be an integral domain with quotient field $K$, and let $J$ be the integral closure of $D$. Then $J$ is a Prüfer domain if and only if $K$ is a P-extension of $D$.

Proof.. Suppose that $K$ is a $P$-extension of $D$. Then $K$ is, $a$ fortiori, a $P$-extension of $J$. We invoke Theorem 2 to conclude that $J$ is a Prüfer domain.

If, conversely, $J$ is a Prüfer domain, then by Theorem $2, K$ is a $P$-extension of $J$ and hence, by Theorem 4 , a $P$-extension of $D$.

There is an extension of Theorem 5 to the case where $K$ is not the quotient field of $D$.

Theorem 6. Let $D$ be a domain with integral closure $J$, and let $L$ be an algebraic extension field of the quotient field $K$ of $D$. Then $J$ is a Prüfer domain if and only if $L$ is a P-extension of $D$.

Proof. If $L$ is a $P$-extension of $D$, then so is $K$, and hence $J$ is a Prüfer domain by Theorem 5. Conversely, if $J$ is a Prüfer domain, and if $t \in L$, then $t$ is a root of a nonzero polynomial $f \in J[X]$. The ideal $A_{f}$ of $J$ is finitely generated, and hence is invertible. If $A_{f}^{-1}=$ $\left(g_{0}, g_{1}, \cdots, g_{n}\right)$, and if $g=\sum_{i=0}^{n} g_{i} X^{i}$ then $A_{f g}=A_{f} A_{g}=J$ so that $f g \in J[X]$ and $(f g)(t)=f(t) g(t)=0$. It follows that $L$ is a $P$-extension of $J$, and hence by Theorem $4, L$ is a $P$-extension of $D$.

\section{REFERENCES}

1. N. Bourbaki, Elements de Mathématique, Algèbre Commutative, Chapitre VII, Diviseurs, Hermann, Paris, 1965.

2. Robert Gilmer, -Multiplicative Ideal Theory, Marcel Dekker, New York, 1972.

3. - Some applications of the Hilfssatz von Dedekind-Mertens, Math. Scand., 20 (1967), 240-244.

4. Robert Gilmer and James A. Huckaba, $\Delta$-rings, J. Algebra, 28 (1974), 414-432.

5. Robert Gilmer and Jack Ohm, Integral domains with quotient overrings, Math. Ann., 153 (1964), 97-103.

6. Irving Kaplansky, Commutative Rings, Allyn and Bacon, Boston, 1970.

7. W. Krull, Beiträge zur Arithmetik Kommutativer Integritätsbereiche. VII. Multiplikativ abgeschlossene Systeme von endlichen Idealen, Math. Z., 48 (1943), 533-552.

8. M. Nagata, Local Rings, Interscience, New York, 1962.

9. Jack Ohn, Integral closure and $(x, y)^{n}=\left(x^{n}, y^{n}\right)$, Monatsh. Math., 71 (1967), 32-39.

10. Hwa Tsang, Gauss' Lemma, University of Chicago Dissertation, 1965.

11. O. Zariski and P. Samuel, Commutative Algebra, Volume II, Van Nostrand, Princeton, N.J., 1960.

Received September 25, 1974. The first author received partial support from National Science Foundation Grant GP-40526 while work on this paper was in progress.

Florida State University 

D. E. Bennett, Strongly unicoherent continua ............................. 1

Walter R. Bloom, Sets of p-spectral synthesis ................................ 7

R. T. Bumby and D. E. Dobbs, Amitsur cohomology of quadratic extensions: Formulas and number-theoretic examples ................. 21

W. W. Comfort, Compactness-like properties for generalized weak topological sums

D. R. Dunninger and J. Locker, Monotone operators and nonlinear biharmonic boundary value problems ...

T. S. Erickson, W. S. Martindale, 3rd and J. M. Osborn, Prime nonassociative algebras

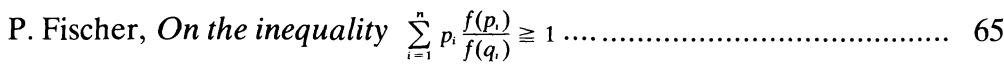

G. Fox and P. Morales, Compact subsets of a Tychonoff set ............... 75

R. Gilmer and J. F. Hoffmann, A characterization of Prüfer domains in terms of polynomials ......................................................... 81

L. C. Glaser, On tame Cantor sets in spheres having the same projection in each direction ......................................................... 87

Z. Goseki, On semigroups in which $X=X Y X=X Z X$ if and only if

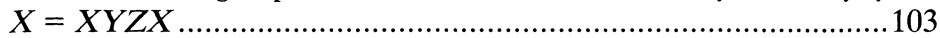

E. Grosswald, Rational valued series of exponentials and divisor

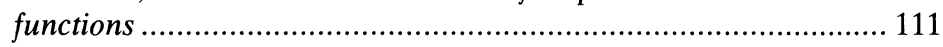

D. Handelman, Strongly semiprime rings ...................................... 115

J. N. Henry and D. C. Taylor, The $\bar{\beta}$ topology for $w^{*}$-algebras ............. 123

M. J. Hodel, Enumeration of weighted p-line arrays .......................... 141

S. K. Jain and S. Singh, Rings with quasiprojective left ideals .............. 169

S. Jeyaratnam, The diophantine equation $Y(Y+m)(Y+2 m) \times$

$$
(Y+3 m)=2 X(X+m)(X+2 m)(X+3 m) \ldots \ldots \ldots \ldots \ldots \ldots \ldots . . .183
$$

$\mathrm{R}$. Kane, On loop spaces without $p$ torsion .........................................189

Alvin J. Kay, Nonlinear integral equations and product integrals ..........203

A. S. Kechris, Countable ordinals and the analytic hierarchy, I ...........223

Ka-Sing Lau, A representation theorem for isometries of $C(X, E) \ldots \ldots . .229$

I. Madsen, On the action of the Dyer-Lashof algebra in $H_{*}(G)$..........235

R. C. Metzler, Positive linear functions, integration, and Choquet's theorem ........................................................................................ 277

A. Nobile, Some properties of the Nash blowing-up ............................297

G. E. Petersen and G. V. Welland, Plessner's theorem for Riesz conjugates 


\section{Pacific Journal of Mathematics}

\section{Vol. 60, No. $1 \quad$ September, 1975}

Donald Earl Bennett, Strongly unicoherent continua ................ 1

Walter Russell Bloom, Sets of p-spectral synthesis ................ 7

Richard Thomas Bumby and David Earl Dobbs, Amitsur cohomology of

quadratic extensions: formulas and number-theoretic examples .......

W. Wistar (William) Comfort, Compactness-like properties for generalized

weak topological sums ...................................

Dennis Robert Dunninger and John Stewart Locker, Monotone operators

and nonlinear biharmonic boundary value problems ..............

Theodore Erickson, Wallace Smith Martindale, III and J. Marshall Osborn,

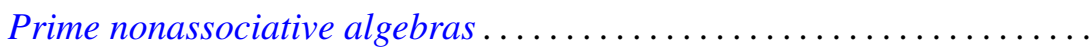

Pál Fischer, On the inequality $\sum_{i=0}^{n}\left[f\left(p_{i}\right) / f\left(q_{i}\right)\right] p_{i} \geq i \ldots \ldots \ldots \ldots \ldots$

Geoffrey Fox and Pedro Morales, Compact subsets of a Tychonoff set.......

Robert William Gilmer, Jr. and Joseph F. Hoffmann, A characterization of

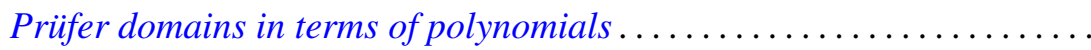

Leslie C. Glaser, On tame Cantor sets in spheres having the same projection

in each direction . ...................................

Zensiro Goseki, On semigroups in which $x=x y x=x z x$ if and only if

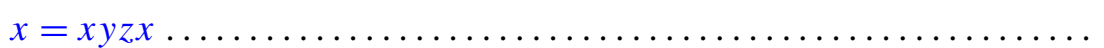

Emil Grosswald, Rational valued series of exponentials and divisor

functions.

David E. Handelman, Strongly semiprime rings

Jackson Neal Henry and Donald Curtis Taylor, The $\bar{\beta}$ topology for

$W^{*}$-algebras

Margaret Jones Hodel, Enumeration of weighted p-line arrays ...

Surender Kumar Jain and Surjeet Singh, Rings with quasi-projective left

ideals.

S. Jeyaratnam, The Diophantine equation

$$
Y(Y+m)(Y+2 m)(Y+3 m)=2 X(X+m)(X+2 m)(X+3 m) \ldots
$$

Richard Michael Kane, On loop spaces without $p$ torsion

Alvin John Kay, Nonlinear integral equations and product integrals ...

Alexander S. Kechris, Countable ordinals and the analytical hierarchy.

$I$.

Ka-Sing Lau, A representation theorem for isometries of $C(X, E)$

Ib Henning Madsen, On the action of the Dyer-Lashof algebra in $H_{*}(G)$

Richard C. Metzler, Positive linear functions, integration, and Choquet's

theorem.

Augusto Nobile, Some properties of the Nash blowing-up

Gerald E. Peterson and Grant Welland, Plessner's theorem for Riesz. 